

Д. А. Зенюк, Г. Г. Малинецкий, Формирование паттернов в системе реакциидиффузии с дробными производными по времени, Матем. моделирование, 2020, том 32, номер 6, 53-65

DOI: https://doi.org/10.20948/mm-2020-06-04

Использование Общероссийского математического портала Math-Net.Ru подразумевает, что вы прочитали и согласны с пользовательским соглашением

http: //www . mathnet.ru/rus/agreement

Параметры загрузки:

IP : 54.162 .85 .209

26 апреля 2023 г., 13:46:38 


\title{
ФОРМИРОВАНИЕ ПАТТЕРНОВ \\ В СИСТЕМЕ РЕАКЦИИ-ДИФФУЗИИ \\ С ДРОБНЫМИ ПРОИЗВОДНЫМИ ПО ВРЕМЕНИ
}

\author{
(C) 2020 г. Д.А. Зенюк, Г.Г. Малинецкий
}

Институт прикладной математики им. М.В. Келдыша РАН, Москва eldrich@yandex.ru

DOI: $10.20948 / \mathrm{mm}-2020-06-04$

На примере абстрактной модели брюсселятора рассмотрены сценарии формирования сложных паттернов в нелинейных средах с диффузией и дифференциальными операторами нецелого порядка. С помощью стандартной техники линейного анализа получены точные выражения для критических значений параметров, при которых в системе наблюдаются неустойчивости определенного типа. Для бифуркации Хопфа и бифуркации коразмерности 2 критерии устойчивости существенно зависят от порядка дробной производной. Предсказания линейной теории подкреплены результатами численного анализа.

Ключевые слова: дробное исчисление, системы реакции-диффузии.

\section{PATTERN FORMATION IN REACTION-DIFFUSION SYSTEM WITH TIME-FRACTIONAL DERIVATIVES}

\section{D.A. Zenyuk, G.G. Malinetsky}

Keldysh Institute of Applied Mathematics, Russian Academy of Science, Moscow

In the present paper possible scenarios of pattern formation in non-linear media with diffusion and differential operators of non-integer order are studied for the abstract Brusselator model. By means of the standard linear analysis exact critical values for different types of instabilities are derived. It is shown that stability criteria significantly depend on the order of the fractional derivative in case of the Hopf and C2TH bifurcations. Predictions of the linear theory are confirmed by numerical simulation.

Key words: fractional calculus, reaction-diffusion systems.

\section{1. Введение}

Дробное интегро-дифференциальное исчисление было создано почти два столетия назад. За это время было предложено несколько неэквивалентных подходов к определению интегралов и производных нецелого порядка, 
детально исследованы их свойства и продемонстрированы связи между некоторыми из этих операторов. Историческую ретроспективу со множеством ссылок, а также строгое изложение основных теоретических результатов можно найти в [1]. Несмотря на свою богатую историю, активное использование этого аппарата для решения различных прикладных задач началось лишь с середины 20 в. Можно выделить два направления исследований, где инструментарий теории дробного интегро-дифференцирования оказался наиболее подходящим - это механика вязкоупругих сред и аномальная диффузия. Изложение вопросов, связанных с первым из этих двух направлений, см. в [2]. Мы же чуть более подробно остановимся на втором.

Термин «аномальная» используется потому, что в этих процессах среднеквадратичные отклонения положения блуждающих частиц нелинейно зависят от времени: $\left\langle x^{2}(t)\right\rangle \sim t^{\gamma}$. Режимы с $0<\gamma<1$ называются субдиффузионными, с $1<\gamma<2$ - супердиффузионными. Обстоятельное перечисление реальных физических процессов, для которых характерна такая аномальная динамика, приведено в [3]. Среди примеров субдиффузии отметим, например, транспорт носителей заряда в аморфных полупроводниках, ядерномагнитный резонанс в пористых и трубчатых средах и рептацию (тепловое движение длинных линейных макромолекул естественного или искусственного происхождения в концентрированных растворах и полимерных расплавах). Одно из возможных объяснений замедления диффузии заключается в том, что в этих системах блуждающие частицы вынуждены тратить время на обход пустот и тупиков, как бы «теряясь» в сложных внутренних переходах внутри среды. Примеры супердиффузии обычно связаны с турбулентным переносом в неравновесных средах, см. ссылки в [4]. Аномальные диффузионные процессы, в свою очередь, являются представителями более широкого класса эволюционных процессов, протекающих в системах со сложной внутренней структурой и нелокальными временными корреляциями, которые объединены по общим названием «странная кинетика» $[4,5]$.

Ниже будут кратко описаны те конструкции дробного исчисления, которые необходимы для дальнейшего изложения. Левосторонние дробные интегралы Римана-Лиувилля порядка $\alpha>0$ на конечном сегменте $[a, b]$ определяются выражением

$$
\left(I_{a+}^{\alpha} f\right)(t)=\frac{1}{\Gamma(\alpha)} \int_{a}^{t} f(\tau)(t-\tau)^{\alpha-1} d \tau, \quad t>a .
$$

Левосторонние производные Капуто имеют вид 


$$
\left(D_{a+}^{\alpha} f\right)(t)=\left(I_{a+}^{\alpha} f^{(n)}\right)(t), \quad \alpha \notin \mathbb{N} \cup\{0\}, \quad\left(D_{a+}^{\alpha} f\right)(t)=f^{(n)}(t), \quad \alpha \in \mathbb{N} \cup\{0\} .
$$

Здесь $n=[\alpha]+1, \alpha \geq 0$ и $[\alpha]$ означает целую часть $\alpha$. У указанных операторов существуют также правосторонние аналоги, отличающиеся положением переменного предела интегрирования и знаками слагаемых внутри интеграла. Основные свойства лево- и правосторонних операторов совпадают. Если переменная, по которой производится дробное интегро-дифференцирование, имеет смысл времени, то только левосторонние операторы будут каузальными: для определения значения в момент времени $t$ они используют лишь предысторию процесса. Поэтому всюду далее мы будем использовать только их.

Существуют и другие, неэквивалентные подходы к определению операторов дробного дифференцирования - в первую очередь это производные Римана-Лиувилля [1]. Разумеется, сразу возникает вопрос, какой из них следует выбрать. В контексте изучения динамических систем нецелого порядка операторы Капуто являются наиболее подходящими. Это связано с двумя их свойствами. Во-первых, производная Капуто от постоянной равна нулю, что позволяет определять стационарность привычным образом. Вовторых, уравнения с операторами Капуто требуют задания начальных условий в терминах обычных производных. Для дифференциальных операторов Римана-Лиувилля это не так - начальные условия также должны быть выражены через дробные производные.

Для дальнейшего изложения нам также понадобится функции МиттагЛеффлера [6, 7]. Это степенные ряды вида

$$
E_{\alpha}(z)=\sum_{k=0}^{+\infty} \frac{z^{k}}{\Gamma(\alpha k+1)}, \quad z \in \mathbb{C}, \quad \operatorname{Re} \alpha>0 .
$$

Функции $E_{\alpha}\left(\lambda t^{\alpha}\right), \lambda \in \mathbb{C}, t>0$ играют ту же роль для оператора Капуто $D_{0+}^{\alpha}$, что и функции $\exp (\lambda t)$ для обычного оператора дифференцирования. Однако, в отличие от обычной экспоненты, они не обладают полугрупповым свойством. Действительно, используя определение (2), можно показать, что

$$
E_{\alpha}\left(\lambda t^{\alpha}\right) E_{\alpha}\left(\mu t^{\alpha}\right)=E_{\alpha}\left((\lambda+\mu) t^{\alpha}\right)
$$

только если $\alpha=1$, либо если хотя бы одно из чисел $\lambda$ и $\mu$ равно нулю. Некоторые вопросы дробного исчисления, опущенные здесь, рассмотрены в [8], там же можно найти более подробную библиографию. 


\section{2. Брюсселятор с дробными производными}

В данном разделе мы подробно рассмотрим следующую модельную двухкомпонентную систему с диффузией и нелинейной химической кинетикой

$$
\begin{aligned}
& \left({ }_{t} D_{0+}^{\alpha} u\right)(t, z)=D_{1} L^{-2} \partial_{z}^{2} u+A-(B+1) u+u^{2} v, \\
& \left({ }_{t} D_{0+}^{\alpha} v\right)(t, z)=D_{2} L^{-2} \partial_{z}^{2} v+B u-u^{2} v .
\end{aligned}
$$

Здесь $L$ - размер расчетной области, $z \in[0,1]$ - безразмерные пространственные координаты, $A, B$ и $D_{i}$ - неотрицательные коэффициенты, $0<\alpha<1$. При $\alpha=1$ она впервые была рассмотрена И. Пригожиным и Р. Лефевером в 1968 г. как идеализированная модель для изучения сложных кооперативных химических процессов в открытых системах и получила название «брюсселятор». Функции $u$ и $v$ задают концентрации двух основных продуктов реакции, коэффициенты $A$ и $B$ - концентрации вспомогательных веществ. Обосновать введение дробных производных в эту систему можно, выполняя предельный переход в одной схеме случайного блуждания - подробное обсуждение этого вопроса см. в [3, 9].

Система (4) при $\alpha=1$ достаточно подробно исследована, см., например, [10-12]. Известно, что она может демонстрировать очень богатый набор динамических режимов, некоторые из которых показаны на рис.1. На этих рисунках черный цвет соответствует наибольшим значениям $u$, а белый - наименьшим. Приведенные здесь решения были получены для начальных условий, соответствующих суперпозиции равновесного решения $(A, B / A)$ и нормального белого шума магнитуды 0.005 .

Базовыми механизмами, формирующими нетривиальные решения, являются здесь неустойчивость Хопфа, приводящая к возникновению пространственно-однородного нестационарного паттерна (рис.1а), и неустойчивость Тьюринга, при которой в системе наблюдается пространственнонеоднородный стационарный паттерн (рис.1b). При определенном выборе параметров возникает т.н. бифуркация коразмерности 2, при которой эти два механизма конкурируют, одновременно нарушая и пространственную, и временную симметрию. В этом режиме можно наблюдать различные паттерны (рис.1с) с более или менее выраженной структурой. Наконец, в системе возможно формирование хаотических режимов (рис.1d). Типичный для систем реакции-диффузии сценарий, при котором возможно наблюдение хаоса, предполагает, что характерная диффузионная длина, которая определяется величинами $\sqrt{D_{i}}$, пренебрежимо мала по сравнению с размером 
Формирование паттернов в системе реакции-диффузии с дробными ...

$L$ самой области. В этом случае система фактически распадается на множество несвязных нелинейных осцилляторов, каждый из которых «занимает» небольшую область пространства.

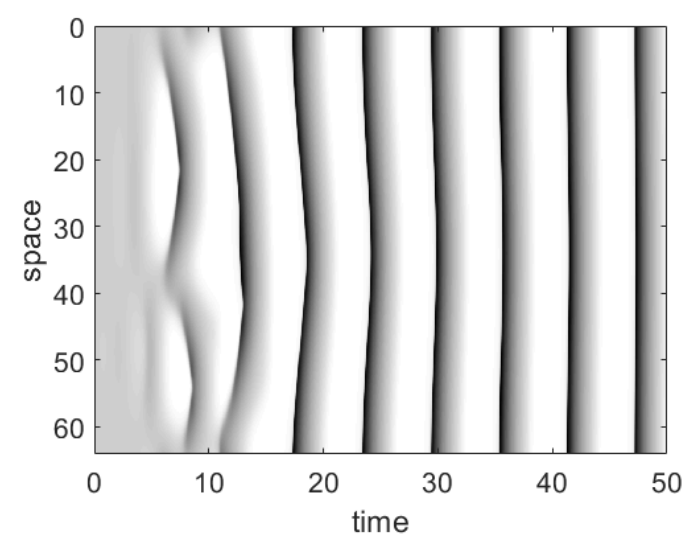

a)

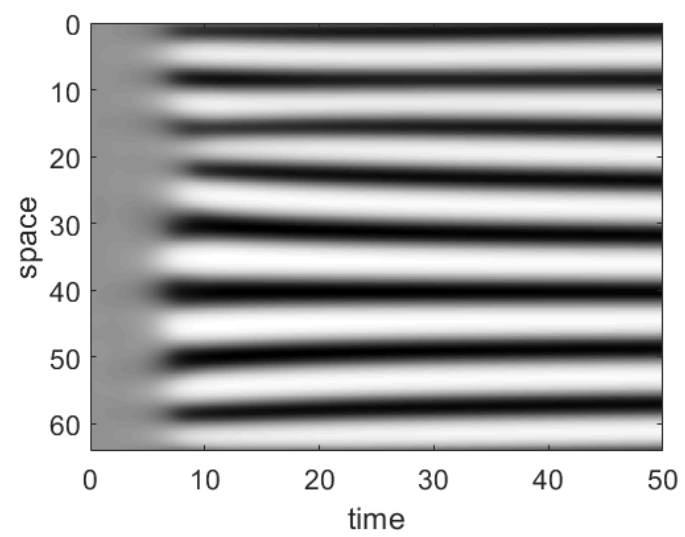

b)


d)

Рис.1. Решения $u$ системы (4) при $\alpha=1: A=2.5, B=9, D_{1}=7, \theta=0.7, L=64$ (a); $A=2, B=4.8, \quad D_{1}=2, \theta=0.2, L=64$ (b); $A=1.118, B=2.75, D_{1}=2$, $\theta=0.2, L=64$ (c); $A=2.5, B=8, D_{1}=4.49, \theta=0.5039, L=512$ (d). Для d использовались однородные условия Неймана, во всех остальных случаях - периодические краевые условия.

Ряд аналитических результатов для системы (4) можно получить с помощью линейной теории. Для определенности будем рассматривать однородные граничные условия Неймана. Следуя стандартной практике, будем считать основным бифуркационным параметром $B$. Для удобства также введем для отношения коэффициентов диффузии обозначение $\theta=D_{1} / D_{2}$.

Легко показать, что единственным положением равновесия системы является решение $u_{0}=A, v_{0}=B / A$. Отклонения от этого пространственнооднородного и стационарного решения, $x_{1}=u-u_{0}, x_{2}=v-v_{0}$, подчиняются уравнениям

$$
\begin{aligned}
& \left({ }_{t} D_{0+}^{\alpha} x_{1}\right)(t, z)=D_{1} L^{-2} \partial_{z}^{2} x_{1}+(B-1) x_{1}+A^{2} x_{2}+B x_{1}^{2} / A+2 A x_{1} x_{2}+x_{1}^{2} x_{2}, \\
& \left({ }_{t} D_{0+}^{\alpha} x_{2}\right)(t, z)=D_{2} L^{-2} \partial_{z}^{2} x_{2}-B x_{1}-A^{2} x_{2}-B x_{1}^{2} / A-2 A x_{1} x_{2}-x_{1}^{2} x_{2} .
\end{aligned}
$$


Граничные условия остаются неизменными. Считая, что возмущения малы по абсолютной величине, мы можем отбросить нелинейные члены и решить полученную линейную систему в явном виде. Действительно, пусть

$$
x_{i}=c_{i} E_{\alpha}\left(\sigma t^{\alpha}\right) \cos \pi m z, \quad m \in \mathbb{N} \cup\{0\}, \quad\left(c_{1}, c_{2}\right)^{\top}=\mathbf{c} .
$$

Легко проверить, что указанная конструкция удовлетворяет граничным условиям. После подстановки этого пробного решения в линейную систему получим, что вектор с с необходимостью должен быть правым собственным вектором матрицы

$$
\left(\begin{array}{cc}
B-1-m^{2} \pi^{2} D_{1} / L^{2} & A^{2} \\
-B & -A^{2}-m^{2} \pi^{2} D_{2} / L^{2}
\end{array}\right),
$$

а $\sigma$ должны удовлетворять характеристическому уравнению

$$
\sigma^{2}+\sigma[b(m)-a(m)]+A^{2} B-a(m) b(m),
$$

где мы использовали обозначения

$$
a(m)=B-1-m^{2} \pi^{2} D_{1} / L^{2}, \quad b(m)=A^{2}+m^{2} \pi^{2} D_{2} / L^{2} .
$$

Устойчивость решения линейной системы с дробными производными зависит от асимптотического поведения функций Миттаг-Леффлера. В рассматриваемом случае для устойчивости требуется, чтобы оба корня $\sigma_{ \pm}$характеристического уравнения были отличны от нуля и модуль их аргумента не превосходил $\alpha \pi / 2$. Более подробное изложение известных результатов по устойчивости динамических систем нецелого порядка можно найти в [13].

Если оба корня действительные, то решение линейной задачи теряет устойчивость лишь в том случае, если один из них становится больше нуля, в то время как другой остается отрицательным. Для этого необходимо выполнение следующих условий:

$$
a(m)-b(m)<0, \quad a(m) b(m)-A^{2} B=0 .
$$

Критическое значение параметра $B$, соответствующее этим условиям, равно

$$
B_{T}(m)=1+\theta A^{2}+\frac{A^{2} L^{2}}{m^{2} \pi^{2} D_{2}}+\frac{m^{2} \pi^{2} D_{1}}{L^{2}} .
$$

Первая бифуркация должна произойти в окрестности минимума

$$
B_{T}(x)=(1+A \sqrt{\theta})^{2}, \quad x^{2}=\frac{A L^{2}}{\pi^{2} \sqrt{D_{1} D_{2}}}
$$


критической кривой, т.е. при целом $m \neq 0$, для которого $B_{T}(m)$ окажется ближе всего к $B_{T}(x)$. Показатель $\alpha$ не играет здесь никакой роли, поэтому результат ничем не отличается от стандартной теории. При бифуркации Тьюринга в решении линейной системы будет доминировать член, пропорциональный $\cos \pi m z$, поскольку $E_{\alpha}(0)=1$, что соответствует формированию стационарного пространственно-неоднородного паттерна.

Комплексные корни возникают лишь при выполнении

$$
[A-\sqrt{d(m)}]^{2}<B<[A+\sqrt{d(m)}]^{2}, \quad d(m)=1+m^{2} \pi^{2}\left(D_{1}-D_{2}\right) / L^{2} .
$$

Необходимым условием существования комплексных корней является неравенство $d(m)>0$.

При $\alpha=1$ решение линейной системы становится неустойчивым, если $a(m)-b(m)=0$, когда корни характеристического полинома будут чисто мнимыми. Критическое значение $B$, при котором это происходит, равно

$$
B_{H}(m, 1)=1+A^{2}+m^{2} \pi^{2}\left(D_{1}+D_{2}\right) / L^{2} .
$$

В случае $\alpha<1$ потеря устойчивости впервые возникает при выполнении условия $\left|\arg \sigma_{ \pm}\right|=\alpha \pi / 2$. Тогда критическое значение $B$ должно быть решением нелинейного уравнения

$$
\operatorname{arctg} \frac{\sqrt{4 A^{2} B-[b(m)+a(m)]^{2}}}{a(m)-b(m)}=\frac{\alpha \pi}{2} .
$$

Положив $\gamma=\operatorname{tg}(\alpha \pi / 2)$, его можно привести к более простому полиномиальному виду

$$
-B^{2}+2 B\left[A^{2}+d(m)\right]-\left[A^{2}-d(m)\right]^{2}=\gamma^{2}\left[B-B_{H}(m, 1)\right]^{2} .
$$

Это уравнение может иметь несколько положительных корней $B_{H}(m, \alpha)$, но лишь один из них удовлетворяет условию $B_{H}(m, \alpha)>B_{H}(m, 1)$. Если это условие не будет выполнено, то $a(m)-b(m)<0$, и тогда, очевидно, $\sigma_{ \pm}$будут иметь отрицательную вещественную часть, а значит, их аргумент гарантированно будет больше $\alpha \pi / 2$, и решение линейной задачи будет устойчиво. Примеры критических кривых $B_{H}(m, \alpha)$ показаны на рис.2.

Если $\alpha=1$, то первая бифуркация Хопфа всегда происходит при $m=0$, поскольку $B_{H}(m, 1)$ монотонно возрастает по $m$. Решение линейной системы будет иметь незатухающие колебания по $t$, но при этом не будет зависеть от $z$. Если же $\alpha<1$, то зависимость $B_{H}(m, \alpha)$ от $m$ оказывается более 
сложной. При $\theta>1$ критические кривые будут напоминать ветвь параболы, направленной вверх. Если $\theta=1$, то критические кривые по-прежнему монотонно возрастают, хотя теперь у них может меняться направление выпуклости. Наконец, при $\theta<1$ меняется не только характер выпуклости, но и монотонность зависимости $B_{H}(m, \alpha)$ - формально возможна ситуация, при которой минимум критической кривой достигается при $m \neq 0$. В этом случае решение линейной системы, теряющее устойчивость, зависело бы и от $t$, и от $z$. Такой механизм неустойчивости не может наблюдаться в стандартной системе, его появление обусловлено наличием производных нецелого порядка. Тем не менее этот гипотетический сценарий не реализуется, потому что еще до того, как эта точка будет достигнута, уже произойдет бифуркация Тьюринга. Для удобства дальнейшего анализа будем считать, что волновое число $m$ может меняться непрерывно. Нетривиальный минимум критических кривых, если он вообще существует, не зависит от $\alpha$ и достигается в точке $y$, где критическая кривая пересекает верхнее ограничение на $B$, гарантирующее существование комплексных корней:

$$
B_{H}(y, \alpha)=[A+\sqrt{d(y)}]^{2} .
$$


Рис.2. Слева: зависимости $B_{H}(m, \alpha)$ от $m$ при нескольких $\alpha$ и $A=2, D_{1}=10, \theta=1.6$, $L=64$. Справа: зависимости $B_{H}(m, \alpha)$ и $B_{T}(m)$ от $m$ при нескольких $\alpha$ и $A=2.5, D_{1}=10, \theta=0.5, L=64$.

После несложных преобразований получим

$$
\sqrt{d(y)}=A\left[\theta-1+\sqrt{(\theta-1)^{2}+4 / A^{2}}\right] / 2 .
$$

Оказалось, что при любых $A$ и $\theta<1$ выполняется неравенство $B_{H}(y, \alpha)>$ $>B_{T}(x)$. Действительно,

$$
A+\sqrt{d(y)}-1-A \sqrt{\theta}>A(\theta+1) / 2-A \sqrt{\theta}=A(1-\sqrt{\theta})^{2} / 2>0 .
$$


Бифуркация коразмерности 2 происходит в том случае, когда параметры подобраны таким образом, что при некотором $m_{*} \neq 0$ выполняется $B_{H}(0, \alpha)=B_{T}\left(m_{*}\right)$. Из этого равенства следует, что между $\theta$ и $A$ существует функциональная связь

$$
A^{2} \theta+2 A \sqrt{\theta}+A^{2}+2 A / \sqrt{1+\gamma^{2}}=0 .
$$

Легко показать, что лишь один корень этого квадратичного относительно $\sqrt{\theta}$ уравнения будет положительным - он равен

$$
\sqrt{\theta_{*}}=\left(\sqrt{1+A^{2}+2 A / \sqrt{1+\gamma^{2}}}-1\right) / A \text {. }
$$

Выполнение условия $\theta<\theta_{*}$ гарантирует, что при последовательном увеличении параметра $B$ первой всегда будет происходить бифуркация Тьюринга, в противном случае - всегда бифуркация Хопфа. Примеры зависимостей $\theta_{*}$ от $A$ при некоторых $\alpha$ показаны на рис.3а. Хорошо видно, что с уменьшением $\alpha$ эти кривые сдвигаются вверх, расширяя область значений $\theta$, в которой можно наблюдать стационарные паттерны Тьюринга.


Рис.3. Слева: зависимости критического отношения коэффициентов диффузии $\theta_{*}$ от $A$ при нескольких $\alpha$. Справа: сравнение решений $u(t)$ системы (4) без диффузии для $\alpha=0.75$ (сверху) и $\alpha=1$ (снизу) с различными близкими начальными условиями, $A=2, B=7$.

Поведение системы в линейном приближении зависит от выбора краевых условий. Мы подробно рассмотрели модель с отсутствием потоков на границах. Можно также рассматривать неоднородные условия Дирихле

$$
u(t, 0)=u(t, 1)=A, \quad v(t, 0)=v(t, 1)=B / A,
$$

и периодические условия. В первом случае в пробном решении пространственная часть задается функцией $\sin \pi m z$, где $m \in \mathbb{N}$. Для периодических ус- 
ловий собственные функции оператора $\partial_{z}^{2}$ будут иметь вид $\exp (2 \pi i m z)$, $m \in \mathbb{N} \cup\{0\}$. Приведенные выше выражения для критических значений $B_{T}(m)$ и $B_{H}(m, \alpha)$ остаются справедливыми с той лишь разницей, что для периодических условий $m$ нужно заменить на $2 m$. Следует, однако, подчеркнуть, что граничные условия могут оказывать существенное влияние на формирование нетривиальных паттернов в системе. Так, условия Дирихле могут подавлять формирование периодических по времени режимов: если параметры выбраны так, что $D_{2}-D_{1}>L^{2} / \pi^{2}$, то условие $d(m)>0$ не может быть выполнено ни при каком $m \in \mathbb{N}$, и собственные числа $\sigma_{ \pm}$не могут быть комплексными.

Результаты линейного анализа интересно сравнить с решениями, полученными непосредственным численным моделированием (см. рис.4). Для этого мы сначала выполнили переход к системе связных дифференциальных уравнений

$$
\begin{aligned}
& \left(D_{0+}^{\alpha} \mathbf{U}\right)(t)=D_{1} h^{-2} L^{-2} M \mathbf{U}+A-(B-1) \mathbf{U}+\mathbf{U} \circ \mathbf{U} \circ \mathbf{V}, \quad \mathbf{U}=\left(U_{1}, \ldots U_{N_{z}}\right)^{\top}, \\
& \left(D_{0+}^{\alpha} \mathbf{V}\right)(t)=D_{2} h^{-2} L^{-2} M \mathbf{V}+B \mathbf{U}-\mathbf{U} \circ \mathbf{U} \circ \mathbf{V}, \quad \mathbf{V}=\left(V_{1}, \ldots V_{N_{z}}\right)^{\top} .
\end{aligned}
$$

Здесь $U_{i}=u\left(t, z_{i}\right), V_{i}=v\left(t, z_{i}\right), z_{i}=i / N_{z}, i=0 \div N_{z}, \mathbf{U} \circ \mathbf{V}$ обозначает покомпонентное умножение векторов одинаковой формы, а $M$ - квадратная матрица, ненулевые элементы которой определяют разностную аппроксимацию дифференциального оператора $\partial_{z}^{2}$. Во внутренних точках использовалась стандартная трехточечная схема, аппроксимация в граничных точках зависит от выбранных краевых условий.

При $\alpha=1$ полученная система обыкновенных дифференциальных уравнений решалась стандартным методом Рунге-Кутты 4-го порядка точности с автоматическим выбором шага. Тестовые расчеты показали, что использование явных схем для нелинейных уравнений нецелого порядка вынуждает использовать очень маленький шаг разбиения по $t$, поэтому мы использовали неявную схему из [14]. Системы нелинейных алгебраических уравнений на каждой итерации решались методом Ньютона-Рафсона с начальным приближением, равным значениям $\mathbf{U}$ и $\mathbf{V}$ с предыдущего временного слоя. Все приведенные в этом разделе численные решения были получены при $N_{z}=2^{9}$, количество шагов по времени в неявной схеме равно $N_{t}=2000$.

Наиболее заметным отличием от стандартной системы является возможность возникновения хаотических режимов. Уже при $\alpha=0.97$ не удалось подобрать параметры так, чтобы наблюдать динамику, похожую на 
pис.1d. Малость коэффициентов диффузии по-прежнему приводит к очень слабому согласованию эволюции отдельных пространственных областей, но соответствующие им осцилляторы уже нельзя считать независимыми. На рис. $3 b$ в качестве примера показано, как меняются решения брюсселятора без диффузии при уменьшении $\alpha$. При $D_{i}=0$ в системе существует предельный цикл для любого $\alpha \leq 1$ : суперкритическая бифуркация Хопфа происходит при $B=B_{H}(0, \alpha)$, когда комплексно сопряженные корни характеристического уравнения пересекают границу области устойчивости. Однако, как видно на рисунке, при $\alpha<1$ поведение решений, у которых начальные состояния мало отличаются, заметно меняется по сравнению со стандартной системой. По-видимому, это связано с «эффектом памяти», характерным для систем дробного порядка: предыстория, которая вносит значительный вклад в динамику, будет почти одинаковой, если начальные состояния достаточно близки. Кроме того, даже слабое диффузионное связывание в этом случае также будет учтено в предыстории, что может выступать в роли дополнительного механизма синхронизации. Поэтому эволюция локальных осцилляторов будет гораздо более согласованной, чем при $\alpha=1$.



a)



b)
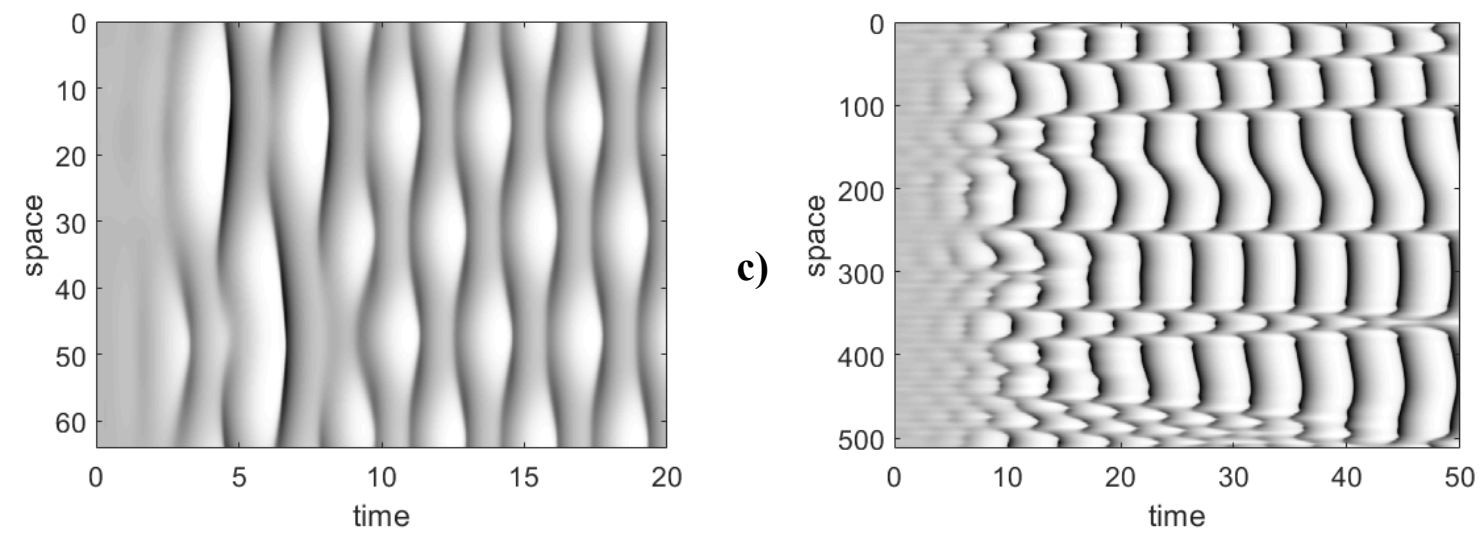

d)

Рис.4. Решения $u$ системы (4) с периодическими граничными условиями: $\alpha=0.8, A=2, B=7, D_{1}=10, \theta=2, L=64$ (a); $\alpha=0.5, A=2$, $B=7, D_{1}=5, \theta=0.6024, L=64$ (b); $\alpha=0.8, A=2, B=7, D_{1}=10$, $\theta=0.65, L=64$ (c); $\alpha=0.98, A=2, B=6, D_{1}=4, \theta=2, L=512$ (d). 


\section{3. Заключение}

Аномальная диффузия, наряду с реологией вязких сред, на сегодняшний день является наиболее строго обоснованным примером применения аппарата дифференциальных операторов нецелого порядка к описанию реальных физических процессов. В стандартных моделях реакции-диффузии формирование сложных пространственно-временных паттернов и возникновение диффузионного хаоса достаточно хорошо изучены и теоретически, и с помощью численного анализа. Для моделей с дробными производными понимание управляющих ими динамических механизмов пока не достигло такого уровня полноты. Поэтому исследования в этой области по-прежнему весьма актуальны.

Применение стандартных методов линейного анализа к брюсселятору - одной из наиболее известных двухкомпонентных моделей - позволило выявить ряд характерных особенностей, связанных с введением дробных производных по временному переменному. Формирование стационарных пространственно-неоднородных паттернов Тьюринга вполне ожидаемо не отличается от стандартной модели с обычными производными. Критические кривые для параметра $B$ в случае бифуркации Хопфа, напротив, демонстрируют существенную зависимость от $\alpha$. Более того, при $\alpha<1$ становится возможным еще один механизм потери устойчивости, когда корни характеристического уравнения пересекают границу области устойчивости при $m \neq 0$ - решение соответствующей линейной задачи будет одновременно зависеть от $t$ и $z$. И хотя в рассмотренном в настоящей работе случае этот механизм не действует, сама его возможность является отличительным свойством дробных систем. Возможно, этот эффект удастся продемонстрировать при рассмотрении модели с другой химической кинетикой.

Критическое значение отношения диффузионных коэффициентов, определяющее, бифуркация какого рода будет первой при последовательном увеличении $B$, также зависит от показателя $\alpha$. Уменьшение $\alpha$ при фиксированном $A$ делает наблюдение паттернов Тьюринга более «простым». Аналогичное наблюдение для несколько отличающейся модели, в которой использовался дифференциальный оператор Римана-Лиувилля, было получено в [15].

Наиболее заметным отличием от стандартной модели является сильное подавление хаотических режимов, возникающих при слабом диффузионном

связывании, когда коэффициенты $D_{i}$ малы по сравнению с $L^{2}$. В этом случае фазы отдельных осцилляторов, на которые распадается система, доста- 
точно хорошо согласованы - это обусловлено эффектом памяти, возникающим из-за дробных производных, показатель которых не слишком близок к единице. Формирующийся в результате паттерн обладает более выраженной структурой и больше похож на те, что возникают в стандартной системе в окрестности бифуркации коразмерности 2.

\section{СПИСОК ЛИТЕРАТУРЫ}

1. S.G. Samko, A.A. Kilbas, O.I. Marichev. Fractional integrals and derivatives: theory and applications. - Yverdon: Gordon \& Breach Science publishers, 1993, $1012 \mathrm{p}$.

2. F. Mainardi. Fractional calculus and waves in linear viscoelasticity. - London: Imperial College Press, 2010, $347 \mathrm{p}$.

3. R. Metzler, J. Klafter. The random walk's guide to anomalous diffusion: a fractional dynamics approach // Physics reports, 2000, v.339, №1, p.1-77.

4. L.M. Zelenyi, A.V. Milovanov. Fractal topology and strange kinetics: from percolation theory to problems in cosmic electrodynamics // Physics-Uspekhi, 2004, v.47, №8, p.749.

5. M.F. Shlesinger, G.M. Zaslavsky, J. Klafter. Strange kinetics // Nature, 1993, v.363, №6424, p.13-37.

6. A.A. Kilbas, H.M. Srivastava, J.J. Trujillo. Theory and applications of fractional differential equations. - Amsterdam: Elsevier, 2006, 541 p.

7. R. Gorenflo et al. Mittag-Leffler functions, related topics and applications. - Berlin: Springer, 2014, $441 \mathrm{p}$.

8. Д.А. Зенюк, Г.Г. Малинеикий. Одномерный брюсселятор с дробными производными по времени // Препринты ИПМ им. М.В. Келдыша, 2019, № 98, 32 с.

D.A. Zeniuk, G.G. Malinetskii. Odnomernyi briusseliator s drobnymi proizvodnymi po vremeni // Preprinty IPM im. M.V. Keldysha, 2019, № 98, 32 s.

9. T.A.M. Langlands, B.I. Henry, S.L. Wearne. Turing pattern formation with fractional diffusion and fractional reactions // J. of Physics: Condensed Matter, 2007, v.19, №6, p.065115.

10. G. Nicolis, I. Prigogine. Self-organization in nonequilibrium systems. - NY: J. Wiley \& Sons, 1977, $512 \mathrm{p}$.

11. A. De Wit. Spatial patterns and spatiotemporal dynamics in chemical systems // Advances in Chemical Physics, 1999, v.109, p.435-514.

12. A. De Wit et al. Spatiotemporal dynamics near a codimension-two point // Physical Review E. 1996, v. 54, №1, p.261.

13. C.P. Li, F.R. Zhang. A survey on the stability of fractional differential equations // The European Physical Journal Special Topics, 2011, v.193, №1, p.27-47.

14. R. Garrappa. Numerical solution of fractional differential equations: A survey and a software tutorial // Mathematics, 2018, v.6, №2, p.16.

15. B.I. Henry, T.A.M. Langlands, S.L. Wearne. Turing pattern formation in fractional activator-inhibitor systems // Physical Review E, 2005, v.72, №2, p.026101.

Поступила в редакцию 28.10 .2019

После доработки 28.10.2019

Принята к публикации 23.12.2019 